\title{
Reorganizar para avançar: a experiência da Atenção Primária à Saúde de Nova Lima/MG no enfrentamento da pandemia da COVID-19
}

Michelle Vieira Fernandez, Dayanna Mary de Castro, Luísa da Matta Machado Fernandes, Isabel Cristina Alves

\section{RESUMO}

Países como o Brasil que possuem a Atenção Primária à Saúde como porta de entrada de um sistema de saúde universal têm o potencial de enfrentar a pandemia da COVID19 de forma transversal e centrada na demanda da comunidade. A experiência do município de Nova Lima apresenta uma reorganização da APS diante da crise sanitária, apoiada nos vínculos estabelecidos no território e na continuidade do cuidado. 0 presente estudo tem o objetivo de descrever essa experiência municipal em ações baseada na garantia do direito à saúde para os usuários.

Palavras-chave: Atenção Primária; Atenção Básica-SUS; COVID-19; sistema de saúde; cuidado integral.

\section{ABSTRACT}

Countries like Brazil that have Primary Health Care as the gateway to a universal health system can face the COVID-19 pandemic with integrated care and focus on community demand. The experience of the municipality of Nova Lima presents a reorganization of PHC in the face of the health crisis, supported by the bonds established in the territory and continuity of care. This study aims to describe this municipal experience based on guaranteeing the health rights for users.

Keywords: Primary Health Care; managed care; health system; COVID-19; comprehensive care.
Revista da Rede APS 2020

Publicada em: 09/06/2020

DOI:10.14295/aps.v2i2.84

Michelle Vieira Fernandez (Universidade de Brasília (UNB), Brasília, DF, Brasil)

Dayanna Mary de Castro Isabel Cristina Alves

(Secretaria Municipal de Saúde de Nova Lima, Nova Lima, MG, Brasil)

Luísa da Matta Machado Fernandes (Instituto Rene Rachou/FioCruz Minas, Belo Horizonte, MG, Brasil)

Correspondência para: Michelle Vieira Fernandez michelle.fernandez@unbl.br

Dayanna Mary de Castro dabnovalima@gmail.com

Luísa da Matta M. Fernandes luisa.fernandes@fiocruzl.br

Isabel Cristina Alves isabelalvesnovalima@gmail.co

Submissão recebida em 28 de maio de 2020.

Aceito para publicação em 08 de junho de 2020 .

Avaliado pelo sistema Double Blind Review com participação dos editores. 


\section{INTRODUÇÃO}

A Atenção Primária à Saúde (APS) é a porta de entrada do sistema de saúde brasileiro. Esse nível de atenção do Sistema Único de Saúde (SUS) tem eficiência comprovada e se caracteriza pelos seguintes atributos: primeiro contato; longitudinalidade; integralidade ou abrangência; coordenação do cuidado; centralidade na família; orientação para a comunidade e competência cultural ${ }^{1}$. Diante dos desafios gerados pela pandemia causada pelo novo coronavírus SARS-Cov-2, trazendo a doença conhecida como COVID-19, países com APS consolidada e como a porta de entrada no sistema de saúde têm a possibilidade de articular o combate à pandemia de forma intersetorial. Nesses países, o combate à epidemia de COVID-19 passa por um enfrentamento comunitário, como é o exemplo da Espanha e Itália ${ }^{2,3,4}$.

Nesse sentido, o Brasil tem uma vantagem em potencial considerando a estruturação da APS no SUS, baseada no território e com equipes multiprofissionais dedicadas a entender as peculiaridades dos lugares de atuação e a nortear $o$ atendimento a partir das características de cada território. A Estratégia de Saúde da Família (ESF) é um dos pilares da organização da APS e hoje tem uma importante capilaridade no território nacional com a cobertura da população alcançando $65,7 \%$,6,7.

No entanto, a resposta brasileira à pandemia vem centrando-se na atenção hospitalar, passando pela criação de novos leitos e compra de respiradores, dando pouca ênfase ao papel da APS. A atuação da APS vem mostrando-se incipiente e descoordenada, principalmente quando se observa o papel desempenhado pelo Ministério da Saúde nesse enfrentamento ${ }^{8}$.

É nesse cenário de crise sanitária, com desvalorização da APS como estratégia para enfrentamento da pandemia, que a gestão da Atenção Básica ( $A B$ ) em Nova Lima, MG, propôs a reestruturação da atenção à população.

\section{NOVA LIMA NO ENFRENTAMENTO DA}

\section{PANDEMIA}

O Minas Gerais é um estado com mais de 21 milhões de habitantes e 823 municípios. Nova Lima é o décimo município mais populoso da região metropolitana de Belo Horizonte ${ }^{9}$. A cidade de 95 mil habitantes, conta com 22 Unidades Básicas de Saúde (UBS) e 25 equipes de Saúde da Família (eSF), que atingem 90,9\% da população ${ }^{7}$. Compõem também a APS três equipes do Núcleo Ampliado de Saúde da Família (NASF) e 07 referências técnicas em saúde mental ${ }^{7}$. O município é marcado por grande desigualdade social, uma vez que conta com número elevado de população de baixa renda e SUS dependente, mas é rodeado por condomínios de alto luxo.

Em 16 de março de 2020 Nova Lima declarou Situação de Emergência no âmbito municipal decorrente da pandemia de COVID-19 ${ }^{10}$, tendo o primeiro caso de contágio confirmado em 18 de março. Em 27 de maio de 2020, o cenário da crise sanitária em Nova Lima apresentava os seguintes dados: 139 casos confirmados para COVID-19, 107 casos recuperados, 909 casos em investigação, 0 óbitos ${ }^{11}$.

No final de março, o número de casos na cidade cresceu mais rápido do que no restante do estado, dado o acesso de parte da população à rede privada e complementar, o que facilitou a testagem. Esse cenário inicial, marcado também pela falta de informações sobre a doença, dificuldade de acesso a equipamentos de proteção individual (EPIs) e desfalque de profissionais na assistência, afastados por serem parte do grupo de risco, colocou o município em estado de alerta. Era necessário a suspensão de algumas atividades para conseguir prestar atendimento seguro, rápido e efetivo a sintomáticos respiratórios.

As agendas dos profissionais foram reorganizadas de forma a ampliar o acesso à demanda espontânea, grupos foram suspensos e consultas eletivas foram bloqueadas. As consultas programadas foram desmarcadas 
após contato da equipe de saúde com o usuário e análise da demanda, mantendo-se apenas a rotina de pré-natal e puerpério. O fluxo de atendimento das equipes foi organizado visando a identificação imediata do sintomático respiratório ainda na recepção da UBS, local diferenciado para a espera da consulta e prioridade no atendimento. Notas técnicas foram elaboradas pela gestão municipal para orientação de fluxos e protocolos de atendimentos e vídeos foram disponibilizados para treinamento quanto ao uso correto dos $\mathrm{EPIs}^{10}$.

Entretanto, a coordenação da $A B$ percebeu que as potencialidades da APS poderiam ser mais exploradas. Por meio da sistematização das informações coletadas nos protocolos do Ministério da Saúde e da Secretaria Estadual de Minas Gerais, discussão entre trabalhadores da assistência e outras secretarias de saúde e videoconferências realizadas pela Associação Brasileira de Saúde Coletiva (ABRASCO) e Rede Unida sobre o tema, foi elaborada a Nota Técnica no 12 para sensibilizar os profissionais da APS quanto à importância da abordagem da determinação social da saúde no enfrentamento da pandemia ${ }^{12}$. O treinamento das equipes utilizou uma metodologia de matriciamento e dispersão. Os gerentes das unidades foram convidados a estudar e a debater a nota técnica para em seguida capacitarem as eSF, NASF e Saúde Mental.

\section{Manejo, vigilância e prevenção}

O manejo clínico do caso suspeito de COVID-19 nas UBS envolve a identificação imediata dos usuários com sintomas respiratórios; a adoção de medidas para evitar contágio; a estratificação da gravidade da Síndrome Gripal; o atendimento dos casos leves; a prescrição e a orientação quanto ao isolamento domiciliar; a estabilização e o encaminhamento dos casos graves a serviços de urgência, bem como a notificação. As equipes se organizaram para realizar o monitoramento de todos os casos suspeitos e confirmados identificados na sua própria unidade, nos outros pontos de atenção da rede referenciados pela Vigilância Epidemiológica (VE) e também pela central telefônica de atendimento (Alô Saúde) implantada no município durante a pandemia.

O contato telefônico para o monitoramento dos casos suspeitos e confirmados é realizado por profissionais da eSF ou NASF no intervalo de 48 ou 24 horas, a depender da avaliação clínica do usuário. As informações obtidas no monitoramento clínico são registradas em prontuário e informadas à VE; estas incluem verificação dos sintomas, seguimento da evolução do quadro e a investigação do surgimento de sintomas nos contatos.

Com o objetivo de identificar e isolar precocemente os casos suspeitos de COVID-19, as equipes realizam busca ativa de sintomáticos respiratórios na comunidade. Os Agentes Comunitários de Saúde (ACS) fazem contato por telefone ou aplicativo de mensagens com as famílias de sua microárea indagando quanto à presença dos sinais e sintomas de gripe. Os pacientes identificados com sintomas gripais são encaminhados para o(a) médico(a) ou enfermeiro(a) da equipe para atendimento telefônico, que determinará a conduta com base no primeiro atendimento.

Idosos, crianças, portadores de doenças crônicas não estabilizados ou de risco alto, imunossuprimidos e gestantes foram classificados como usuários do grupo de risco e são monitorados com maior frequência. Instituições de longa permanência para idosos, unidades penitenciárias e casas de acolhimento institucional também são foco de monitoramento intensivo. Para os casos que o contato remoto não é possível, o ACS realiza a visita domiciliar. Para auxiliar no contato com os pacientes, cada equipe recebeu um smartphone, além do telefone fixo e um celular que já existia na UBS. Cada unidade também foi equipada com duas webcams, que foram instaladas em computadores já existentes.

\section{Educação em saúde}

Por meio de grupos de aplicativo de mensagens e vídeos produzidos pelos profissionais do Núcleo de Apoio à Saúde da Família à 
comunidade recebe orientações sobre a pandemia, autocuidado, hábitos de vida saudáveis, arboviroses e demais necessidades de saúde. As ações de educação em saúde já existiam nas unidades antes mesmo da crise sanitária atual. No entanto, essas atividades eram realizadas de forma presencial. Essas novas modalidades de interação foram criadas para a nova conjuntura de crise sanitária.

Além disso, os ACSs continuam com a abordagem das pessoas nas ruas e em espaços comunitários oferecendo orientações acerca da COVID-19 e da importância do isolamento social. Estão também vigilantes para a identificação de estabelecimentos que não estejam seguindo as determinações dos Decretos Municipais, acionando a Vigilância Sanitária Municipal quando necessário. Toda a equipe está apta a identificar no território as principais barreiras da população local para seguir as recomendações dadas, bem como mobilizar a rede de apoio necessária para superá-las. Para a execução dessas atividades, foi disponibilizado material informativo sobre a COVID-19 e uma nota técnica orientando os profissionais sobre o protocolo de atuação.

O trabalho articulado com a secretaria de comunicação de Nova Lima garantiu a adesão da população ao isolamento domiciliar e as equipes conseguiram focar na reestruturação da demanda das unidades. Ganharam tempo para ter acesso aos EPIs e à confiança dos trabalhadores, que passaram a enxergar seu papel central no combate à COVID-19 e perceberam um crescimento de casos dentro do esperado inicialmente, sem apresentar crescimento descontrolado da curva de contaminação. Nesse momento, a coordenação municipal percebeu a oportunidade de retomar a essência da APS, com foco nos grupos mais vulneráveis e mantendo a continuidade do cuidado.

\section{Suporte aos grupos mais vulneráveis}

As equipes foram orientadas a identificar, dentro de seu território, os usuários de maior vulnerabilidade, como: povos e comunidades tradicionais; pessoas que vivem em assentamentos precários; população em situação de rua; beneficiários de programas sociais; pessoas com deficiência e idosos sem apoio social; pacientes com comorbidades e autocuidado insuficiente; e mulheres e crianças vítimas de violência doméstica. Após a identificação, cada equipe desenvolveu abordagem e suporte a esses usuários, com o envolvimento de outros atores sociais e também dos serviços do Sistema Único de Assistência Social, considerando os recursos e especificidades de seu território.

Entre as estratégias desenvolvidas encontramos: orientações específicas sobre a COVID-19 e distribuição de máscaras para a população em situação de rua; confecção de máscaras para a população de baixa renda; orientações à população quanto aos benefícios sociais a que têm direito; mobilização da rede de apoio na comunidade para idosos e doentes crônicos com autocuidado insuficiente e sem rede de suporte social ou familiar; e abordagem individualizada do ACS no acompanhamento de mulheres com histórico de violência doméstica.

\section{Acompanhamento das condições crônicas}

O plano de cuidado às condições crônicas na APS em tempos de COVID-19 foi estabelecido para evitar a descontinuidade do cuidado e consequente agravamento de condições de saúde pré-existentes. $O$ plano está estruturado em cinco etapas:

1. Levantamento do público-alvo: idosos; crianças menores de um ano; gestantes; puérperas; hipertensos; diabéticos; portadores de transtorno mental; pacientes com feridas crônicas; demais patologias de curso longo (HIV/Aids, tuberculose, etc.) ou outras condições de importância epidemiológica na área de abrangência da UBS. As equipes da APS identificam tais usuários a partir do cadastro individual e domiciliar, relatórios operacionais do registro clínico eletrônico (eSUS-AB) e registro/controle manual dos ACS. 
2. Seleção dos grupos prioritários: a equipe, a partir das características da população local, patologias e condições mais prevalentes e grupos de maior risco na área de abrangência da UBS, define os grupos prioritários para início do monitoramento/atendimento. Algumas equipes optaram por iniciar o acompanhamento por grupos de faixa etária ou comorbidade; outras identificaram os grupos de maior risco para cada condição.

3. Contato telefônico inicial para identificação das demandas: as equipes se organizaram para realizar uma ligação prévia para identificação das demandas/necessidades de cada paciente. Esse contato inicial é feito pelos ACS, apoiados também pelos técnicos e auxiliares de enfermagem e profissionais do NASF/Saúde Mental. As abordagens são breves, acolhedoras e humanizadas, os profissionais perguntam sobre sinais e sintomas da COVID-19, hábitos de vida atuais e algumas questões relacionadas à condição crônica do paciente.

4. Identificação dos pacientes para teleconsultas: a partir das informações obtidas no contato telefônico inicial, as equipes selecionam os pacientes com indicação de teleatendimento.

5. Teleconsultas: as consultas são feitas por médicos(as), enfermeiros(as), profissionais do NASF e Saúde Mental, com a utilização de recursos tecnológicos, dispositivos móveis e equipamentos de acesso remoto. A renovação de receita é realizada à distância desde que o médico na teleconsulta seja o mesmo que já acompanha o usuário e com pelo menos uma consulta presencial prévia. A prescrição médica é entregue ao representante designado pelo usuário, evitando a presença desse na UBS. Caso o usuário tenha uma frágil rede de suporte social, a receita é entregue na sua residência pelo ACS da área. Todo o atendimento é registrado em prontuário. Após o teleatendimento, se o profissional julgar que é necessária a avaliação presencial do paciente, realiza-se, preferencialmente, uma visita domiciliar. Se não for possível, a consulta presencial é agendada na UBS, com o devido cuidado para evitar aglomerações na unidade.

\section{Aprendizados no caso de Nova Lima:}

1. Necessidade de manter o cuidado continuado dos pacientes adscritos à UBS: A suspensão das consultas eletivas e atividades coletivas nas UBS, necessária para conter a propagação da COVID-19, pode trazer sérias consequências para pacientes e para o sistema de saúde caso não haja novas estratégias para acompanhamento efetivo e oportuno desses grupos. Usuários vulneráveis não devem sofrer redução do acompanhamento previsto nos protocolos e a classificação de risco definida em prioridade pela equipe de saúde é uma estratégia fundamental para o sucesso do combate à pandemia.

2. Importante priorizar as ações de acordo com a vulnerabilidade da população: os grupos vulneráveis são diversos e cada equipe da APS, conhecedora do seu território pode identificar e priorizar as ações utilizando dos recursos disponíveis na comunidade. Assim, passamos a ter um cuidado focado no território e não apenas em demandas individuais.

3. Valorização dos profissionais da APS: A consistência do trabalho da gestão, demostrando o papel da APS no enfrentamento da pandemia apoiando a estratégia na construção de vínculos no território. Os profissionais relataram se sentirem reconhecidos e representados, diminuindo a ansiedade e trazendo assertividade ao trabalho. 
4. Participação do Controle Social: Como foi descrito, o engajamento da população para o sucesso do isolamento social é fundamental, o que aconteceu em Nova Lima. No entanto, cabe aqui uma reflexão sobre a necessidade de avançar no planejamento da reestruturação do processo de trabalho nas UBSs. Inicialmente o conselho municipal não foi envolvido no planejamento. Reconhecendo a centralidade da participação do controle social no SUS, a coordenação da $A B$ organizou reuniões com os conselheiros para informar, debater e discutir após a publicação das notas técnicas. Avalia-se que as reuniões deveriam ter sido implementadas no início do planejamento com o intuito de aumentar a adesão da comunidade.

A única chance de o Brasil diminuir a transmissibilidade e enfrentar o vírus sem um colapso no sistema de saúde é investindo no fortalecimento da APS, garantindo a longitudinalidade do cuidado, incorporando tecnologias de teleatendimento e monitoramento à distância, bem como protegendo os profissionais de saúde ${ }^{13}$, assim como exemplificado na experiência de Nova Lima. Monitoramento ativo, ênfase no território, estratégias alternativas de consulta online e proteção dos profissionais de saúde são questões estruturais para o enfretamento da crise $^{14,15,16}$.

O aumento de barreiras de acesso aos cuidados primários de saúde durante a pandemia pode levar a um aumento de $60 \%$ de mortes maternas e um incremento de 18 a $23 \%$ na mortalidade infantil ${ }^{17}$. Nesse contexto, é essencial a continuidade do cuidado das ações da APS, em especial para as populações vulneráveis e de alto risco, com medidas de proteção social focalizadas ${ }^{18,19}$. Para isso, os profissionais de cada eSF são os mais capacitados pela habilidade que possuem de identificar esses usuários em seu território. $A$ coordenação da $A B$ em Nova Lima compreendeu a centralidade desse foco e implementou políticas locais, mesmo que essas não fossem fomentadas pelo nível de governo central.

\section{CONSIDERAÇÕES FINAIS}

A proposta de reorganização dos processos de trabalho nas UBS de Nova Lima tem permitido o fortalecimento das ações de enfrentamento ao novo coronavírus sem, contudo, perder a essência do que é o cuidado da atenção primária à saúde no município. De forma geral, os profissionais da APS têm tido boa aceitação das novas metodologias do cuidado impostas pela pandemia. No mesmo sentido, o retorno dos usuários também tem sido positivo, refletindo em maior valorização da equipe e fortalecimento do vínculo.

Acessibilidade, integralidade, transversalidade e disponibilidade de bens e serviços na saúde são fundamentais no atual contexto de crise sanitária ${ }^{20}$. Essas diretrizes podem ser identificadas na estratégia implementada por Nova Lima. Sistematizar as experiências locais e potencializar os aprendizados torna-se extremamente importante em um contexto mundial no qual os sistemas de saúde como o do Brasil se mostraram pouco preparados para absorver o impacto de uma pandemia. A pandemia e a necessidade de isolamento social, no entanto, não podem ser causadores da privação do direito ao acesso universal à saúde. É fundamental que os desafios impostos pela pandemia sejam enfrentados dentro do escopo da garantia do direito à saúde. 


\section{NOTAS E REFERÊNCIAS}

1. Starfield B, Shi L, Macinko J. Contribution of Primary Care to Health Systems and Health. Johns Hopkins Bloom Sch Public Heal Milbank Q. 2005;83(3):457-502.

2. Lorenzo SM. La pandemia COVID-19: lo que hemos aprendido hasta ahora desde España. Rede Pesquisa em Atenção Primária à Saúde. 2020;2(1):28-32.

3. WHO-China Joint Mission. Report of the WHO-China Joint Mission on Coronavirus Disease 2019 (COVID-19) [Internet]. WHO. 2020. Available from: https://www.who.int/docs/defaultsource/coronaviruse/who-china-joint-mission-on-covid-19-final-report.pdf

4. Tasca R, Massuda A. Estratégias para reorganização da Rede de Atenção à Saúde em resposta à Pandemia COVID-19: a experiência do Sistema de Saúde Italiano na região de Lazio. Aps Em Rev. 2020;2(1):20-7.

5. Paim JS, Travassos C, Almeida C, Bahia L, Maclnko J. The Brazilian health system: History, advances, and challenges. Lancet [Internet]. 2011;377(9779):1778-97. Available from: http://dx.doi.org/10.1016/S0140-6736(11)60054-8

6. Paim JS. Desafios para a Saúde Coletiva no Século XXI. 2006. 154 p.

7. Brasil. Cobertura da Atenção Básica. Ministério da Saúde. Departamento de Saúde da Família. [Internet]. 2020. Available from: https://egestorab.saude.gov.br/index.xhtml

8. Fernandez M. Como as políticas de saúde deveriam ser conduzidas na pandemia. Nexo Jornal [Internet]. 2020;22-3. Available from: https://www.nexojornal.com.br/ensaio/debate/2020/Como-as-políticas-desaúde-deveriam-ser-conduzidas-na-pandemia

9. IBGE. CENSO 2010 [Internet]. CENSO 2010 - 2019 [cited 2019 Jun 6]. p. 1-2. Available from: https://www.ibge.gov.br/apps/snig/v1/index.html?loc=0

10. Nova Lima. Nota Técnica $\mathbf{N}^{\circ} 07$ (23 de março de 2020) - Protocolo de Manejo Clínico do Coronavírus (Covid-19) na Atenção Primária à Saúde de Nova Lima/MG. 2020 p. 1-26.

11. Minas Gerais. Distribuição dos casos de COVID-19 | Secretaria de Estado de Saúde de Minas Gerais SES [Internet]. Secretaria Estadual de Saúde. 2020. p. 2020. Available from: https://www.saude.mg.gov.br/coronavirus/painel

12. Nova Lima. Nota Técnica $\mathbf{N}^{\circ} 12$ (27 de abril de 2020) - Atenção Primária à Saúde em tempos de Covid19: estratégias para a reorganização dos processos de trabalho nas Unidades Básicas de Saúde. 2020 p. $1-21$.

13. Vitória ÂM, Campos GW de S. ABRASCO: Só com APS forte o sistema pode ser capaz de achatar a curva de crescimento da pandemia e garantir suficiência de leitos UTI. 2020.

14. Engstrom E, Melo E, Giovanella L, Mendes A, Grabois V, Mendonça MHM de. Recomendações para a organização da Atenção Primária à Saúde no SUS no enfrentamento da COVID-19 - OBSERVATÓRIO COVID-19. 2020.

15. Massuda A, Malik AM, Cintra W, Junior F, Neto GV. Pontos-chave para Gestão do SUS na Resposta à Pandemia COVID-19 - Nota técnica n. 6 do Instituto de Estudos para Políticas de Saúde. São Paulo; 2020. 
16. Fernandez $M$, Lotta $G$. Atenção básica e profissionais de saúde: a ponta de lança no combate à epidemia do Covid-19. Jornal Estado de São Paulo - Blogs [Internet]. 2020; Available from: https://politica.estadao.com.br/blogs/gestao-politica-e-sociedade/atencao-basica-e-profissionais-desaude-a-ponta-de-lanca-no-combate-a-epidemia-do-covid-19/

17. Roberton T, Carter ED, Chou VB, Stegmuller A, Jackson BD, Tam Y, et al. Early Estimates of the Indirect Effects of the Coronavirus Pandemic on Maternal and Child Mortality in Low- and Middle-Income Countries. SSRN Electron J [Internet]. 2020;(20):1-8. Available from: http://dx.doi.org/10.1016/S2214109X(20)30229-1

18. Tavares AB, Silveira F, Paes-Sousa R. Proteção Social e COVID-19: a resposta do Brasil e das maiores economias da América Latina. Rev NAU Soc. 2020;11(20):111-29.

19. Barra RP, Morae EN de, Jardim AA, Oliveira KK de, Bonati PC de R, Issa AC, et al. A importância da gestão correta da condição crônica na Atenção Primária à Saúde para o enfrentamento do Covid-19 em Uberlândia, Minas Gerais. 2020;17-9.

20. OEA. Guía Práctica de Respuestas Inclusivas y con Enfoque de Derechos ante el COVID-19 en las Américas. Washington D.C.; 2020. 